\title{
Microwave dielectric loss in oxides: Theory and experiment
}

\author{
Xavi Aupi, Jonathan Breeze, Neboysha Ljepojevic, Lawrence J. Dunne, Nishil Malde, \\ Anna-Karin Axelsson, and Neil McN. Alford ${ }^{\text {a) }}$ \\ Physical Electronics and Materials, Faculty of Engineering, London South Bank University, \\ 103 Borough Road, London SE1 OAA, United Kingdom
}

(Received 2 January 2003; accepted 14 July 2003)

\begin{abstract}
We present a model that provides a description of the microwave dielectric loss in oxides. The dielectric loss $\left(\tan \delta\right.$ ) in single crystal and polycrystalline $\mathrm{MgO}$ and $\mathrm{Al}_{2} \mathrm{O}_{3}$ is measured over the temperature range 70-300 K. We are able to model the dielectric loss in terms of a two-phonon difference model. There are two key parameters in this model: The third derivative, $\phi_{3}$, of the lattice potential and the linewidth, $\gamma$, of the thermal phonons. In polycrystalline samples, rather than considering the different mechanisms of extrinsic loss, it is assumed that the main effect of extrinsic factors is a modification of the linewidth of the thermal phonons. By varying $\gamma(T)$, it is shown that the model can describe the loss in both single crystals and polycrystallines materials. In single crystal and polycrystalline $\mathrm{MgO}$, we use $\gamma$ as a fitting parameter. In single crystal and polycrystalline $\mathrm{Al}_{2} \mathrm{O}_{3}$, we obtain $\gamma(T)$ by Raman spectroscopy. The theory gives the right order of magnitude of the measured loss. () 2004 American Institute of Physics. [DOI: 10.1063/1.1605810]
\end{abstract}

\section{INTRODUCTION}

One of the most persistent problems in materials science is that of dielectric loss. In 1897, Lord Rayleigh published a paper on dielectric waveguides ${ }^{1}$ and, in 1909 , Debye described dielectric spheres. ${ }^{2}$ In 1939, Richtmyer ${ }^{3}$ made experimental measurements on dielectric resonators. With the rapid growth in both terrestrial and satellite communications, dielectric resonators play a key role in future systems. There are three key properties that characterize dielectric resonators. These are the relative permittivity which determines the size of the resonator, the temperature coefficient of resonant frequency which determines the frequency stability, and the dielectric loss which determines the selectivity and performance of the device. Predicting the dielectric loss in real materials at microwave communications frequencies has proven to be highly problematic. This article provides a method for predicting the dielectric loss (the $\tan \delta$ ) in microwave dielectric materials.

The dielectric loss was measured by Rubens and Hertz in 1912 and, ten years later, Ewald ${ }^{5}$ suggested that its origin was the anharmonic interaction between the radiation and the thermal lattice vibrations. When the electromagnetic field is coupled with the lattice vibrations, a mutual perturbation results as in the coupling of any two oscillatory systems. At microwave field frequencies, the coupling of the field with the lattice vibrations is far from resonance. However, the mutual perturbation is still noticeable in the fact that some mechanical lattice vibrations acquire a small fraction of the energy of the electromagnetic field. Then, due to the thirdand higher-order terms in the potential energy of the lattice (which couple one vibrational mode with another), this small fraction of extra energy gradually diffuses into the rest of modes in the lattice and ultimately appears as heat.

\footnotetext{
${ }^{a)}$ Electronic mail: alfordn@sbu.ac.uk
}

The exchange of energy and momentum between lattice vibrational modes is described in terms of phonon-phonon interactions. Gurevich and Tagantsev ${ }^{6-8}$ reported the contribution to the total loss from three- and four-phonon processes). By using the symmetry considerations described by Herring, ${ }^{9-11}$ this theory is able to give a prediction for the intrinsic losses in the different crystal classes. The different crystal classes have different dispersion diagrams, and only in those places where branches are sufficiently close, touch, or intersect, is microwave absorption allowed due to energy conservation rules. For the photon absorption to occur by means of a three-phonon process, the energy gap between phonon branches needs to be smaller than, or of the order of, the energy of the field photons. However, because of the finite lifetime of the phonons, the gap between phonon branches is not well defined and cannot be determined with a higher precision than $h$ times the inverse of the thermal phonon lifetime. In the model by Sparks et al. ${ }^{12}$ such relaxation of the energy conservation constraint due to the poor definition of the energy gap is introduced explicitly in terms of a Lorentzian broadening of the branches. Losses are then calculated in terms of two-phonon difference processes between acoustic and optic branches and are expressed as a function of the linewidth $(\gamma)$ of the Lorentzian line shape. In this article, we report loss measurements as a function of temperature in single crystal and polycrystalline $\mathrm{MgO}$ and $\mathrm{Al}_{2} \mathrm{O}_{3}$. These experimental data are then interpreted in terms of the Sparks et al. ${ }^{12}$ formulas, where the linewidth $\gamma$ of the phonons at the zone boundary, which is temperature dependent, is used either as a fitting parameter $(\mathrm{MgO})$ or is approximated in terms of the linewidth at the zone center directly measured by Raman spectroscopy $\left(\mathrm{Al}_{2} \mathrm{O}_{3}\right)$.

Sparks et al. ${ }^{12}$ build on earlier work by Stolen and Dransfeld. ${ }^{13}$ Stolen and Dransfeld ${ }^{13}$ observed, in some alkaline halides, that the absorption for two-phonon difference processes does not occur for transitions between longitudinal 

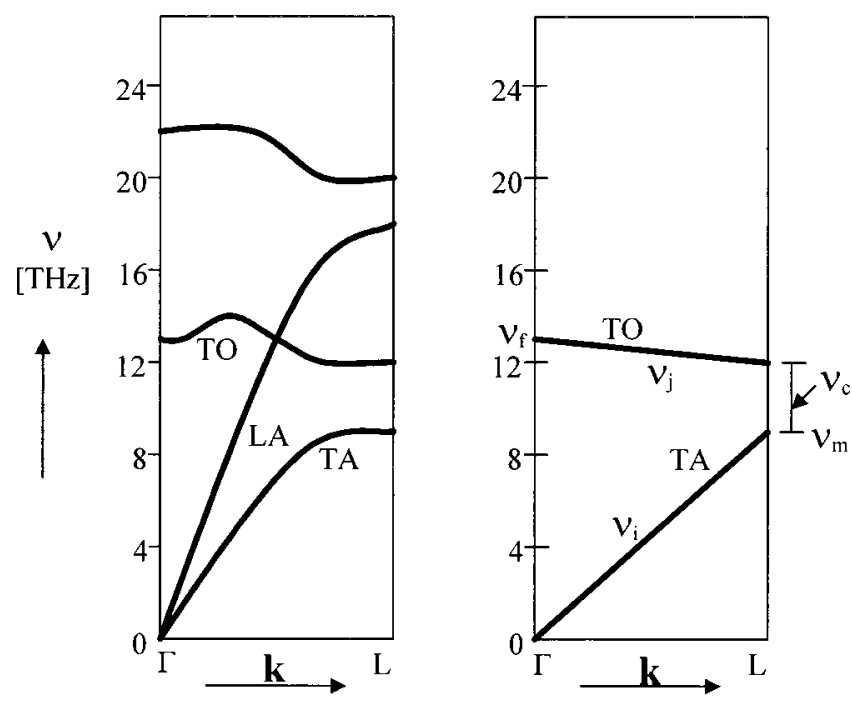

FIG. 1. Left-hand side: Schematic representation of the $\mathrm{MgO}$ dispersion diagram along the $\xi, \xi, \xi$ direction showing various phonon branches. Righthand side: TA and TO branches have been approximated as straight lines (see Ref. 12). The frequency values $\omega_{i}, \omega_{j}, \omega_{f}, \omega_{m}$, and $\omega_{c}$ in [Eq. (9)] are $2 \pi$ times $\nu_{i}, \nu_{j}, \nu_{f}, \nu_{m}$, and $\nu_{c}$, respectively.

and transverse phonons. On the other hand, Eldridge ${ }^{14}$ produced general rules for significant transitions in addition to those allowed by group theory. In the $\mathrm{NaCl}$ structure, Eldridge $^{14}$ shows that transitions are not allowed at the $X$ point, but are at the $L$ point. This might be the reason why, in the Sparks et al. ${ }^{12}$ model, only the transitions between the transverse-acoustic (TA) and transverse-optic (TO) branches at the $L$ point are considered. Contributions coming from other interband processes (such as those between acoustic branches or higher-frequency branches) are not considered although they are not forbidden by the model. Their contribution is assumed to be smaller because either they happen to be in regions where the density of states is low or they are sparsely populated at these temperatures (in the case of higher bands).

The theory by Sparks et al. ${ }^{12}$ is simple. For instance, the dispersion diagram is assumed to be of the type shown in Fig. 1. Then, the dispersion is approximated in three dimensions using a spherical symmetric integral $4 \pi k^{2} d k$. Such an approach makes the model applicable only to simple crystalline structures, such as the alkaline halides, or also, as in the case in the present article, to $\mathrm{MgO}$ and $\mathrm{Al}_{2} \mathrm{O}_{3}$ (the latter is assumed to be not highly anisotropic). These two crystals have, in the $[\xi \xi \xi]$ direction, a dispersion relation similar to that of Fig. 1. The symmetry considerations, shown by Gurevich and Tagantsev ${ }^{6-8}$ to play a decisive role in the estimation of the dielectric loss, are replaced by the measured dispersion relations.

\section{THEORIES VERSUS MEASUREMENTS}

Comparing theoretical predictions of loss ${ }^{6-8,12}$ with $\tan \delta$ measurements is not straightforward because theories assume defect-free single crystals, whereas the measurements are performed on real crystals, which are never defect free. Defects, impurities, or any other departure from symmetry pro- duce additional contributions to the losses, sometimes referred to as extrinsic losses. These contributions are particularly noticeable at low temperatures in single crystals and can be readily observed at room temperature in polycrystalline materials. Polycrystalline materials are of great interest for their use as dielectric resonators in microwave communications applications. Measurements by Alford et al. ${ }^{15,16}$ show that the current theories do not properly describe the losses in polycrystalline materials, since the losses turn out to be dominated mainly by impurities and processing conditions. Even in high-quality single crystals, the measured losses are orders of magnitude greater than those predicted by theory. ${ }^{6-8}$ However, despite difficulties in their application to real materials, we stress that the theories ${ }^{6,7,8,12}$ provide a lower limit for the loss.

\section{SINGLE CRYSTALS AND POLYCRYSTALLINE ANALOGUES}

In order to estimate the dielectric loss in both single crystals and polycrystalline ceramics, we have extended the approximation due to Sparks et al. ${ }^{12}$ We assume that the fact that the linewidth $\gamma$ appears explicitly in the formula provided by the model by Sparks et al. ${ }^{12}$ allows the determination of the loss not only in single crystals but also in their polycrystalline analogues. This is based on the fact that the main effect induced by extrinsic factors is an alteration of the lifetime of the thermal phonons rather than a direct absorption of the microwave photon. Any defect, understood as a breakdown of symmetry, interferes with the passage of the thermal phonons. We found experimental evidence for this in temperature dependent Raman spectroscopy in single crystal sapphire and polycrystalline alumina (see Fig. 4). Note that the temperature dependence of the linewidth of the Reststrahlen mode $\left(418 \mathrm{~cm}^{-1}\right)$ is different in the two cases. Such a difference is due to the major concentration of extrinsic factors in the polycrystalline sample. Although these measurements are not performed at the zone boundary but at the zone center $(k=0)$, we assume that the phonons at the zone boundary are equally affected by the concentration of extrinsic factors, such that the linewidth in the single crystal is still lower than that in the polycrystalline. In the absence of experimental values for $\gamma$ at the zone boundary in $\mathrm{MgO}$, we will use $\gamma$ as a fitting parameter to match the experimental data in single crystal and polycrystalline $\mathrm{MgO}$. We will see that the order of magnitude for $\gamma$ is reasonable. In $\mathrm{Al}_{2} \mathrm{O}_{3}$, we will use the Raman measurements of $\gamma(T)$ at the zone center to estimate the loss in both single crystal sapphire and polycrystalline alumina. In this case, we will note that the difference between their respective values of the linewidth is directly related to their different loss values.

Apart from the linewidth $\gamma$ of the thermal phonons, the loss in this model is also a function of another crucial parameter: The third derivative of the lattice potential $\left(\phi_{3}\right) \gamma$ and $\phi_{3}$ are two key parameters because they are the only anharmonic parameters appearing in the formula [see Eq. (9)]. $\phi_{3}$ is the third-order variation of the interaction energy between atoms associated with a displacement of the atoms from their equilibrium sites. Such a displacement can be performed in several different ways, as many as the number of 
different vibrational modes in the lattice plus linear combinations of them. Thus, each vibrational mode has its related value of $\phi_{3} . \phi_{3}$ can be measured in some cases. For instance, in acoustic modes of long wavelength, the value of $\phi_{3}$ is equal to that of the third-order elastic constants, ${ }^{11}$ which can be obtained experimentally. In our calculations, to obtain the losses in $\mathrm{MgO}$ and $\mathrm{Al}_{2} \mathrm{O}_{3}$, we require $\phi_{3}$ values related to the TA branch at the zone boundary and to the TO of lowest frequency at the zone boundary, too. However, as a first approximation, we use the measured value of the thirdorder elastic constant $\left(\mathrm{C}_{111}\right)$ as an order of magnitude estimate for both modes. In the two crystals under consideration, the $\mathrm{C}_{111}$ is an order of magnitude greater than the other thirdorder elastic constants. Thus, the $\mathrm{C}_{111}$ is considered to be the dominant one. The $\mathrm{C}_{111}$ for both $\mathrm{MgO}$ and $\mathrm{Al}_{2} \mathrm{O}_{3}$ has been measured experimentally. ${ }^{17,18}$ Unlike the linewidth $\gamma$, we assume that the $\mathrm{C}_{111}$ does not vary much from the single crystal to its polycrystalline analogue, provided that the material is not highly anisotropic.

\section{METHODS: EXPERIMENT}

The measurements were performed in a manner similar to that described by Alford et al. ${ }^{16}$ Single crystals of $\mathrm{MgO}$ and $\mathrm{Al}_{2} \mathrm{O}_{3}$ were obtained from ESCETE BV (Enschede, Netherlands) in the form of cylinders of $10 \mathrm{~mm}$ diameter and $4 \mathrm{~mm}$ height. Sintered ceramics of similar dimension were made in our laboratories from fine $\mathrm{MgO}$ or $\mathrm{Al}_{2} \mathrm{O}_{3}$ powder. The powder was uniaxially pressed in a stainless-steel die at a pressure of $100 \mathrm{MPa}$ and then sintered in air at $1650^{\circ} \mathrm{C}$ for between 1 and $6 \mathrm{~h}$. This was sufficient to obtain a sintered disk with near theoretical density. The microwave measurements were performed on an Agilent HP8720C Vector network analyzer with $1 \mathrm{~Hz}$ resolution using either a $30 \mathrm{~mm}$ or $36 \mathrm{~mm}$ diameter oxygen free, high-purity, copper cavity. The height of the cavity was $16 \mathrm{~mm}$. The $\mathrm{TE}_{01 \delta}$ fundamental mode was used for measurement in transmission. This mode was used because of the achievable high electric energy filling factor and low conductor losses. The samples were placed inside the cavity on top of a low-loss quartz support to further reduce the conduction losses. We also used a small diameter aluminum oxide post to support the samples. In order to avoid vibration noise, the support post was glued to the cavity base and the dielectric resonator was in turn glued to the support by means of an adhesive (Opticlean, Dantronix, Platteville). We were able to make an adjustment to the dielectric loss by noting the contribution to the $\tan \delta$ of the adhesive over the temperature range required. The assembly was placed on the cold head of a two-stage GiffordMcMahon cryocooler (Cryophysics, Abingdon, U.K.) which operates over the temperature range of $10 \mathrm{~K}-320 \mathrm{~K}$. The frequency, insertion loss, and loaded $Q$ were measured at small temperature intervals. The radial mode matching technique ${ }^{19}$ was used to calculate the relative permittivity $\epsilon_{r}$ and loss tangent $\tan \delta$ from these data, as well as the temperature coefficient of resonant frequency. Raman spectra were taken with a Renishaw Raman microscope (system
2000), the excitation source was a $488 \mathrm{~nm} \mathrm{Ar}^{+}$laser, and the sample was placed on a temperature stage capable of cooling to $77 \mathrm{~K}$.

\section{MODELING THE $\tan \delta$ AT $f=10 \mathrm{GHz}$ AS A FUNCTION OF TEMPERATURE}

The Sparks et al. ${ }^{12}$ model provides an expression for the relaxation frequency $\Gamma$ of the fundamental Reststrahlen transverse-optical phonon. In this section, we obtain an expression for the $\tan \delta$ as a function of $\Gamma$ by means of the classical damped harmonic oscillator model.

Within the model of the additive damped harmonic oscillators for an isotropic crystal lattice, the dielectric function $\epsilon(\Omega)$ is given by ${ }^{20}$

$$
\epsilon(\Omega)=\epsilon_{\infty}+\frac{4 \pi n e^{* 2}}{M_{r}} \frac{1}{\omega_{\mathrm{TO}}^{2}-\Omega^{2}-i 2 \omega_{\mathrm{TO}} \Gamma_{\mathrm{TO}}(\Omega)},
$$

where $\epsilon_{\infty}$ is the high-frequency dielectric constant, $M_{r}$ is the reduced mass of the unit cell, $n$ is the number of cells per unit volume, $e^{*}$ are the Born effective charges, $\omega_{\mathrm{TO}}$ is the angular frequency of the zero-wave vector of the TO phonon, $\Omega$ is the angular frequency of the ac field, and $\Gamma_{\mathrm{TO}}$ is the imaginary part of the proper self-energy of the TO phonon (or also the relaxation frequency), evaluated at the frequency $\Omega$ of the electromagnetic radiation.

From expression (1), for field frequencies $\Omega$ much lower than the Reststrahlen frequency $\omega_{\mathrm{TO}}$, i.e., $\Omega \ll \omega_{\mathrm{TO}}$, the $\tan \delta$ is

$$
\tan \delta=\frac{\epsilon_{I}}{\epsilon_{R}}=\frac{\epsilon(0)-\epsilon_{\infty}}{\epsilon(0)} \frac{2 \Gamma}{\omega_{T O}},
$$

where $\epsilon_{R}$ and $\epsilon_{I}$ are the real and imaginary part of the dielectric constant given by Eq. (1) and $\epsilon(0)$ is $\epsilon_{R}(\Omega)$ at $\Omega=0$, i.e., the static dielectric constant. Under the assumption that $\mathrm{Al}_{2} \mathrm{O}_{3}$ is not highly anisotropic, we will also use the expression above to relate the $\tan \delta$ and $\Gamma$ in $\mathrm{Al}_{2} \mathrm{O}_{3}$.

\section{RELAXATION FREQUENCY $\Gamma$ FOR MgO}

We now use the Sparks et al. ${ }^{12}$ model to evaluate the relaxation frequency $\Gamma_{\text {TO }}$, hereafter simply denoted by $\Gamma$. To be consistent with their notation, let the notation of $\omega_{\mathrm{TO}}$ be changed to $\omega_{f}$. Then, the relaxation frequency is ${ }^{12}$

$$
\Gamma \approx \frac{2}{\pi} \Gamma_{0}\left[\tan ^{-1} \frac{\omega_{f}-\Omega}{\gamma}-\tan ^{-1} \frac{\omega_{c}-\Omega}{\gamma}\right],
$$

where

$$
\begin{aligned}
\Gamma_{0}= & \frac{1.5 g\left(k_{0}, \Omega\right)}{2 h \nu_{1}} \equiv \Gamma_{\infty}\left(n\left(\omega_{i}\right)-n\left(\omega_{i}+\Omega\right)\right) \\
& \times\left[\frac{k_{0}}{k_{B Z}}\right]^{2}\left[\frac{\omega_{m}^{3}}{\omega_{i} \omega_{j}\left(\omega_{f}-\omega_{c}\right)}\right] \times \sin ^{2} \pi k_{0} / 2 k_{B Z} \\
\Gamma_{\infty}= & \frac{\pi N_{B} h \phi_{3}^{2}}{6 M_{r} M_{<} M_{>} \omega_{f} \omega_{m}^{3}} .
\end{aligned}
$$

The frequencies $\omega_{i}, \omega_{j}, \omega_{c}, \omega_{m}$, and $\omega_{f}$ are $2 \pi$ times the $\nu_{i}, \nu_{j}, \nu_{c}, \nu_{m}$, and $\nu_{f}$ values shown schematically in Figs. 

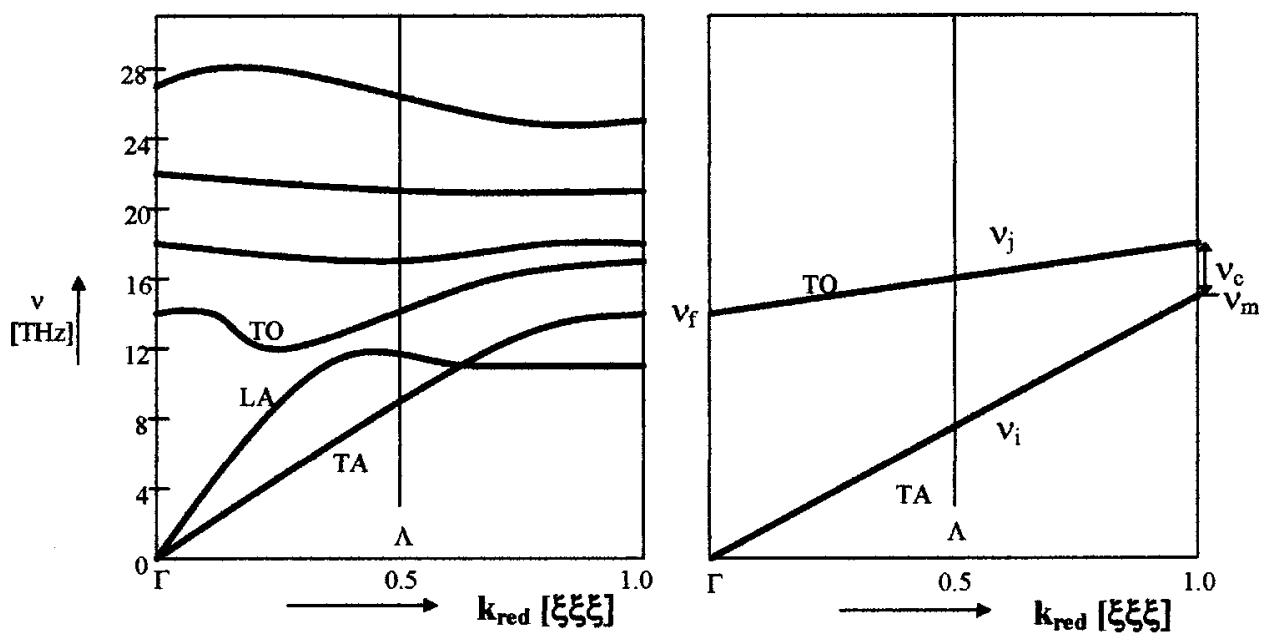

FIG. 2. Left-hand side: Schematic representation of the $\mathrm{Al}_{2} \mathrm{O}_{3}$ dispersion diagram along the direction of three-fold symmetry. Right-hand side: TA and TO branches have been approximated as straight lines.

1 and 2. The wave vector $k_{B Z}$ is also defined there. $\gamma$ is an average of the linewidth of the two modes with frequencies $\omega_{m}$ and $\omega_{c}$, respectively. The frequencies $\omega_{j}$ and $\omega_{i}$ are taken to be $\mathrm{be}^{12}$

$$
\omega_{j}=\omega_{m}+\Omega \text { and } \omega_{i}=\omega_{m}
$$

since $\Omega \ll \omega_{c}$. The $n\left(\omega_{m}\right)=\left[\exp \left(\hbar \omega_{m} / k_{B} T\right)-1\right]^{-1}$ is the Bose-Einstein distribution function. For microwave frequencies, the expression $n\left(\omega_{i}\right)-n\left(\omega_{i}+\Omega\right)$ appearing in Eq. (4), is approximated as ${ }^{12}$

$$
n\left(\omega_{i}\right)-n\left(\omega_{i}+\Omega\right) \approx \frac{\hbar \Omega}{k_{B} T} n\left(\omega_{i}\right)\left[n\left(\omega_{i}\right)+1\right],
$$

where $N_{B}$ is the number of pairs of branches contributing to the relaxation, which is $N_{B}=1$ for both $\mathrm{MgO}$ and $\mathrm{Al}_{2} \mathrm{O}_{3}$. The $M_{<}$and $M_{>}$are the masses of the nearest-neighbor atoms, in this case of $\mathrm{MgO}$, those of oxygen and aluminum. $M_{r}$ is the reduced mass of the unit cell, i.e., $M_{r}^{-1}=M_{<}^{-1}$ $+M_{>}^{-1}$. The $k_{0}$ is ${ }^{12}$

$$
k_{0}=k_{B Z}
$$

since $\Omega<\omega_{c}$ is largely satisfied. The temperature dependence of the third derivative of the lattice potential $\phi_{3}$ is neglected as a first approximation. By introducing the considerations (5) - (7) into the $\Gamma$ expression [Eqs. (3) and (4)], the following is obtained:

$$
\begin{aligned}
\Gamma \approx & \frac{h \phi_{3}^{2}}{3 M_{r} M_{\mathrm{Mg}} M_{\mathrm{O}} \omega_{f}} \frac{1}{\omega_{m}\left(\omega_{m}+\Omega\right)\left(\omega_{f}-\omega_{c}\right)} \frac{\hbar \Omega}{k_{B} T} n\left(\omega_{m}\right) \\
& \times\left[n\left(\omega_{m}\right)+1\right]\left[\tan ^{-1} \frac{\omega_{f}-\Omega}{\gamma}-\tan ^{-1} \frac{\omega_{c}-\Omega}{\gamma}\right]
\end{aligned}
$$

Now, by recovering expression (2) for $\Gamma$, the $\tan \delta$, as a function of temperature is

$$
\begin{aligned}
\tan \delta \approx & \frac{\epsilon(0)-\epsilon_{\infty}}{\epsilon(0)} \frac{2 h \phi_{3}^{2}}{3 M_{r} M_{<} M_{>} \omega_{f}^{2}} \frac{1}{\omega_{m}\left(\omega_{m}+\Omega\right)\left(\omega_{f}-\omega_{c}\right)} \\
& \times \frac{\hbar \Omega}{k_{B} T} n\left(\omega_{m}\right)\left[n\left(\omega_{m}\right)+1\right] \\
& \times\left[\tan ^{-1} \frac{\omega_{f}-\Omega}{\gamma}-\tan ^{-1} \frac{\omega_{c}-\Omega}{\gamma}\right],
\end{aligned}
$$

which is the expression to be plotted as a function of temperature. It stands for the contribution to loss tangent in $\mathrm{MgO}$ coming from those two-phonon difference processes between the TA and TO branches in the $[\xi \xi \xi]$ direction in $k$ space. All of the input values are given in Table I. As stated above, the values of the third derivative $\phi_{3}$ of the lattice potential are assumed to be equal to the measured third-order elastic constant $\mathrm{C}_{111} \cdot{ }^{11}$ In Ref. 21 , the $\phi_{3}$ values are determined using a numerical method. For $\mathrm{MgO}$, we use the value for $\mathrm{C}_{111}$ measured by Bogardus (see table VIII in Ref. 17) in single crystal $\mathrm{MgO}$. The corresponding values for $\omega_{c}, \omega_{m}$, and $\omega_{f}$ are extracted from the phonon dispersion relation along the $[\xi \xi \xi]$ direction reported by Bilz et $a .^{22}$ Masses are derived from the atomic weight of 23.4 for $\mathrm{Mg}, 16$ for $\mathrm{O}$. The reduced mass $\left(M_{r}\right)$ of the $\mathrm{MgO}$ unit cell was calculated as $M_{r}^{-1}=M_{\mathrm{Mg}}^{-1}+M_{\mathrm{O}}^{-1}$. The static and high-frequency dielectric constants $\epsilon(0)$ and $\epsilon_{\infty}$ are approximately 9.8 and 2.9. ${ }^{23}$ In Eq. (9), we use $\gamma$ at room temperature as a fitting parameter to match the loss tangent measurements in both single crystal and polycrystalline $\mathrm{MgO}$.

\section{RELAXATION FREQUENCY $\mathrm{\Gamma}$ FOR $\mathrm{Al}_{2} \mathrm{O}_{3}$}

In sapphire, we consider the measured dispersion relation in the direction of three-fold symmetry. ${ }^{24}$ The corresponding dispersion diagram is approximated as in Fig. 2. The Sparks et al. ${ }^{12}$ model provides, in this case, a formula similar to Eq. (9). Input values are given in Table I.

For the value of $\phi_{3}$, we use the value of the third-order elastic constant $\mathrm{C}_{111}$ measured by Srinivasan et al. ${ }^{18}$ on poly- 
TABLE I. Values for $\mathrm{MgO}$ and $\mathrm{Al}_{2} \mathrm{O}_{3}$ [used in Eq. (9)].

\begin{tabular}{|c|c|c|}
\hline Parameter & $\mathrm{MgO}$ & $\mathrm{Al}_{2} \mathrm{O}_{3}$ \\
\hline $\begin{array}{l}\omega_{m}: \text { Frequency of the transverse acoustic phonon } \\
\text { at the Brillouin zone boundary. }\end{array}$ & $2 \pi \times 12.8 \times 10^{12} \mathrm{~Hz}$ & $2 \pi \times 11.3 \times 10^{12} \mathrm{~Hz}$ \\
\hline $\begin{array}{l}\omega_{c}: \text { Gap between the optical mode and the acoustic } \\
\text { mode at the zone boundary. }\end{array}$ & $2 \pi \times 0.5 \times 10^{12} \mathrm{~Hz}$ & $2 \pi \times 4.6 \times 10^{12} \mathrm{~Hz}$ \\
\hline$\omega_{f}:$ Reststrahlen frequency. & $2 \pi \times 12 \times 10^{12} \mathrm{~Hz}$ & $2 \pi \times 12.5 \times 10^{12} \mathrm{~Hz}$ \\
\hline$\Omega$ : Frequency of the ac field. & $9 \times 10^{9} \mathrm{~Hz}$ & $\begin{array}{l}8.2 \times 10^{9} \mathrm{~Hz} \text { single crystal } \\
9 \times 10^{9} \mathrm{~Hz} \text { polycrystalline }\end{array}$ \\
\hline$\epsilon(0)$ : Static permittivity. & 9.8 & 11.6 \\
\hline$\epsilon_{\infty}$ : High-frequency permittivity. & 2.95 & 3.0 \\
\hline$\phi_{3}:$ Third derivative of the lattice potential. & $-4.9 \times 10^{12} \mathrm{~J} \mathrm{~m}^{-3}$ & $-4.6 \times 10^{12} \mathrm{~J} \mathrm{~m}^{-3}$ \\
\hline$M_{\mathrm{Mg} / \mathrm{Al}}:$ Mass of $\mathrm{Mg} / \mathrm{Al}$ & $M_{\mathrm{Mg}}=4.03 \times 10^{-26} \mathrm{~kg}$ & $M_{\mathrm{Al}}=4.48 \times 10^{-26} \mathrm{~kg}$ \\
\hline$M_{r}:$ Reduced mass. & $1.6 \times 10^{-26} \mathrm{~kg}$ & $3.17 \times 10^{-27} \mathrm{~kg}$ \\
\hline$\gamma$. Linewidth of the phonons at the zone boundary. & $\begin{array}{l}\text { Fitting parameter in single crystal } \\
\text { and polycrystalline } \mathrm{MgO} \text {. }\end{array}$ & $\begin{array}{c}A=1.51 \times 10^{11} \mathrm{~Hz}, B=-2.22 \times 10^{6} \mathrm{~Hz} \mathrm{~K}^{-1}, \\
\text { and } C=2.27 \times 10^{5} \mathrm{~Hz} \mathrm{~K}^{-2} \text { for single crystal. } \\
A=1.215 \times 10^{11} \mathrm{~Hz} \text { and } B=2.5 \times 10^{8} \mathrm{~Hz} \mathrm{~K}^{-1} \text { for polycrystalline. }\end{array}$ \\
\hline
\end{tabular}

crystalline $\mathrm{Al}_{2} \mathrm{O}_{3}$. The frequency values $\omega_{f}, \omega_{c}$, and $\omega_{m}$ are taken in this case from those reported by Bialas et al. ${ }^{24}$ along the direction of three-fold symmetry. Masses are derived from the atomic weight of 16 for $\mathrm{O}$ and 27 for $\mathrm{Al}$. For $\mathrm{Al}_{2} \mathrm{O}_{3}$, where there are four aluminum and six oxygen atoms per unit cell, the reduced mass $M_{r}$ is calculated as the sum of the reciprocals of all atom masses, i.e., $M_{r}^{-1}=4 M_{\mathrm{Al}}^{-1}$ $+6 M_{\mathrm{O}}^{-1}$. The high-frequency and static dielectric constants are 3.0 (Ref. 23) and 11.6. ${ }^{25}$ The values of the linewidth $\gamma(T)$ at the zone boundary are estimated in terms of the measured $\gamma(T)$ at the zone center (see Fig. 4). Note from Fig. 4 that $\gamma(T)$ in sapphire can be approximated as a quadratic function of temperature; $\gamma(T)=A+B T+C T^{2}$, whereas in alumina, a linear regression $\gamma(T)=A+B T$ is sufficient. The values of $A, B$, and $C$, in both single crystal sapphire and polycrystalline alumina, are given in Table I.

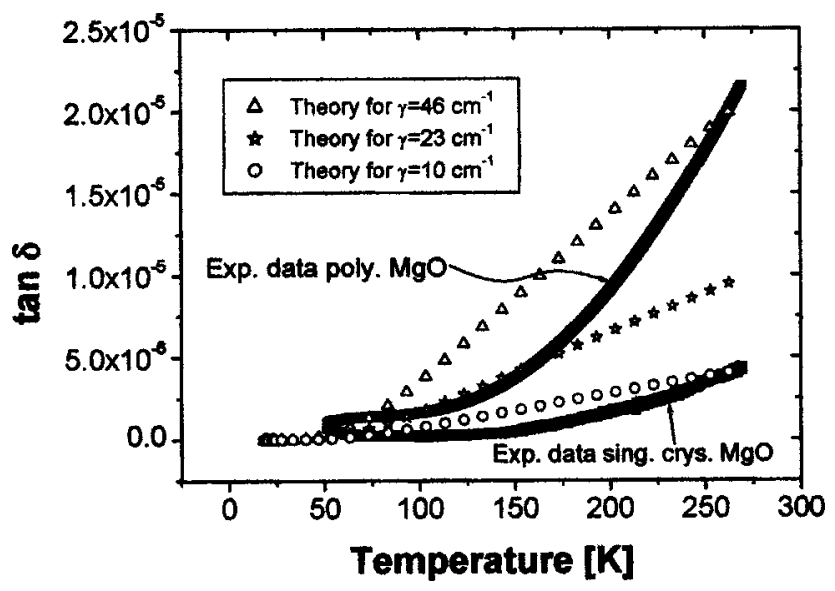

FIG. 3. $\tan \delta$ as function of temperature in single crystal and polycrystalline $\mathrm{MgO}$ at $9 \mathrm{GHz}$. The linewidth $\gamma$ at room temperature is used as a fitting parameter.

\section{RESULTS AND DISCUSSION}

\section{A. $\mathrm{MgO}$}

The slopes of the $\tan \delta$ measured as functions of temperature of single crystal and polycrystalline $\mathrm{MgO}$ in the 70-300 K temperature range show different $T$ dependences as can be seen in Fig. 3. Losses in the polycrystalline sample increase more rapidly with $T$. This difference in behavior is due to extrinsic factors, such as impurities, random grain orientation, defects, etc. We interpret both slopes in terms of Eq. (9), where $\gamma$ is used as a fitting parameter (see Fig. 3). The temperature dependence of $\gamma$ is not included. Only the value at room temperature is considered. This is the reason why the theoretical curves are straight lines rather than monotonic functions of the temperature. In Fig. 3, note that for a value of $\gamma=46 \mathrm{~cm}^{-1}$, the model gives the right order of magnitude of the measured loss in polycrystalline $\mathrm{MgO}$ at room temperature. For a value of $\gamma=10 \mathrm{~cm}^{-1}$, the formula gives the correct value of the measured loss in single crystal $\mathrm{MgO}$. These values need to be compared to those in literature. For instance, in $\mathrm{Si}$, the linewidth of the lowest TO mode at room temperature is between 7 and $18 \mathrm{~cm}^{-1}$ depending on the point at $k$ space. $^{26}$ In Ge, it ranges ${ }^{26,27}$ from 3 to $13 \mathrm{~cm}^{-1}$. In $\mathrm{LiF}, \mathrm{it}^{12}$ is $37 \mathrm{~cm}^{-1}$. On the other hand, from Eq. (2.18) in the Sparks et al. paper, ${ }^{12}$ one can estimate the value of the linewidth in $\mathrm{MgO}$ at room temperature from a linear scaling of the value of the linewidth in $\mathrm{NaCl}$. Using this method, one obtains $\gamma=49 \mathrm{~cm}^{-1}$. Note that the obtained $\gamma$ values by fitting are of the same order of magnitude. However, we do not know whether the increase of $\gamma=10 \mathrm{~cm}^{-1}$ to $\gamma=46 \mathrm{~cm}^{-1}$ from the single crystal to the polycrystalline is realistic.

\section{B. $\mathrm{Al}_{2} \mathrm{O}_{3}$}

From temperature dependent Raman spectroscopy in the single crystal $\mathrm{Al}_{2} \mathrm{O}_{3}$, we obtained $\gamma(T)=A+B T+C T^{2}$ for 


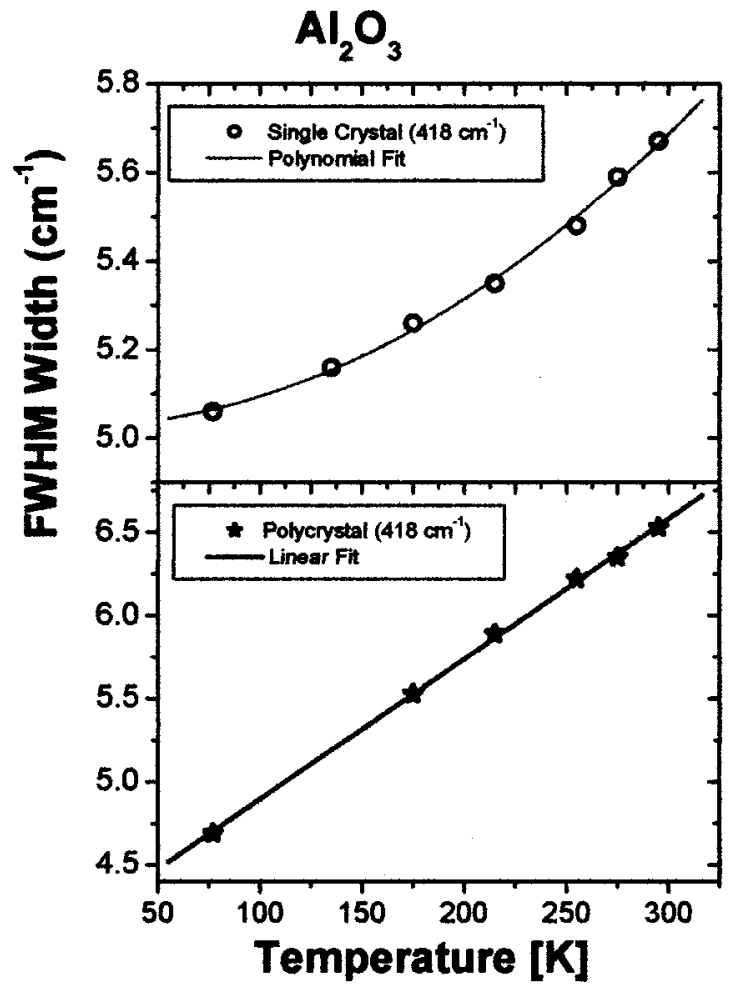

FIG. 4. Temperature dependence of the linewidth of the $418 \mathrm{~cm}^{-1}$ mode (Reststrahlen mode) in single crystal and polycrystalline $\mathrm{Al}_{2} \mathrm{O}_{3}$ obtained from temperature dependent Raman spectroscopy. Polynomial fit $\dot{\gamma}(T)=A$ $+B T+C T^{2}$ in the single crystal and linear fit $\gamma(T)=A+B T$ in the polycrystalline. The values of the parameters are given in Table I.

the $418 \mathrm{~cm}^{-1}$ mode, with $A=1.51 \times 10^{11} \mathrm{~Hz}, B=-2.11$ $\times 10^{4} \mathrm{~Hz} \mathrm{~K}^{-1}$, and $C=2.27 \times 10^{5} \mathrm{~Hz} \mathrm{~K}^{-2}$. For polycrystalline $\mathrm{Al}_{2} \mathrm{O}_{3}$, we obtained $\gamma(T)=A+B T$ with $A=1.215$ $\times 10^{11} \mathrm{~Hz}$ and $B=0.25 \times 10^{9} \mathrm{~Hz} \mathrm{~K}^{-1}$ (see Fig. 4). When these data are substituted into Eq. (9), we obtain the results plotted in Fig. 5. Note that at room temperature, the theory gives the measured loss in both single crystal sapphire and polycrystalline alumina. We consider this to be a satisfactory result. At room temperature, the difference in loss from the

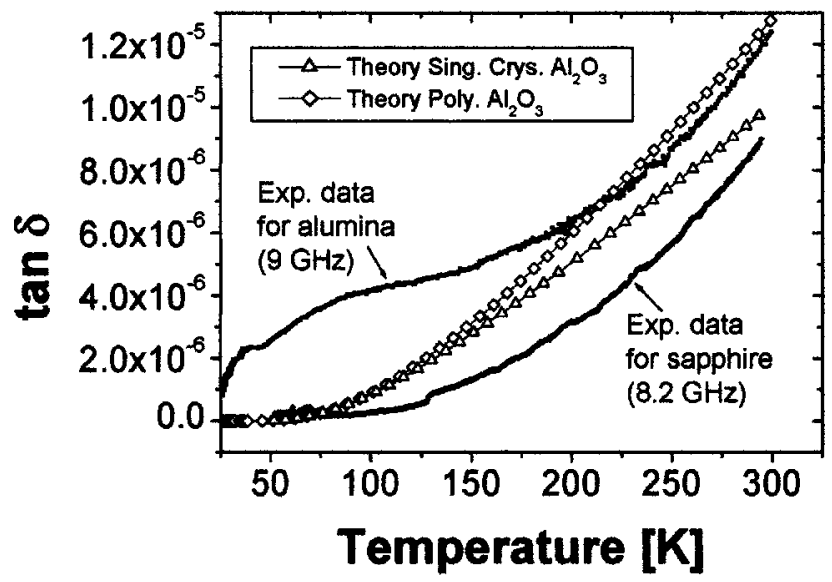

FIG. 5. The measured $\tan \delta$ as function of temperature in single crystal $\mathrm{Al}_{2} \mathrm{O}_{3}$ at $8.2 \mathrm{GHz}$ and polycrystalline $\mathrm{Al}_{2} \mathrm{O}_{3}$ at $9 \mathrm{GHz}$ together with the plot of Eq. (9) for different values of the linewidth $\gamma$ measured by Raman spectroscopy. single crystal sapphire to polycrystalline alumina is well reproduced by the model. Note that the experimental curve for the loss in polycrystalline alumina has a particular shape (especially at low temperatures) that cannot be reproduced by Eq. (9). This suggests the existence of extrinsic mechanisms of absorption in which the Reststrahlen phonons are not involved. Also note that the exact temperature dependence of the loss in both alumina and sapphire is not well reproduced by the theory. This can be easily attributed to the great simplicity of the model (mainly due to the fact that the density of states is not directly taken into account), ${ }^{12}$ which is only expected to give an order of magnitude estimate of the measured loss.

\section{SUMMARY AND CONCLUSIONS}

The temperature dependence of the $\tan \delta$ in both single crystal and polycrystalline $\mathrm{MgO}$ and $\mathrm{Al}_{2} \mathrm{O}_{3}$ was examined in the temperature range of 70-300 K. In order to model these results, we have used a theory developed originally by Sparks et al. ${ }^{12}$ to describe microwave absorption in alkaline halides in terms of two-phonon difference processes. There are two key parameters in this theory: The third derivative $\phi_{3}$ of the lattice potential and the linewidth $\gamma$ of the thermal phonons. We extended the theory to certain oxides with dispersion relations similar to those of the alkaline halides in order to describe the contribution of the TA-TO transitions at the $L$ point in $k$ space to the dielectric loss at microwave frequencies.

We assumed that the main effect brought by the extrinsic factors present in both single crystals and polycrystalline analogues was to increase the linewidth of the thermal phonons. Experimental evidence for this was provided by Raman spectroscopy on single crystal and polycrystalline alumina in the temperature range of $77-300 \mathrm{~K}$, where the linewidth of the Reststrahlen mode in single crystal sapphire exhibited a different temperature dependence than that observed in polycrystalline alumina. However, these measurements were performed at the zone center $(k=0)$ rather than at the zone boundary, where they may be lower. ${ }^{26,27}$

In the case of $\mathrm{MgO}$, we used $\gamma$ in Eq. (9) as a fitting parameter to describe the loss in both single crystal and polycrystalline $\mathrm{MgO}$. As a fitting parameter, $\gamma$ turned to be in the right order of magnitude, i.e., a few $\mathrm{cm}^{-1}$, although their exact values should be checked in future experiments.

In $\mathrm{Al}_{2} \mathrm{O}_{3}$, as a first approximation we used the measured values of $\gamma(T)$ at the zone center. In this case, the theoretical expression does not contain any adjustable parameter and gives the right order of magnitude of the measured loss in both sapphire and alumina in the 70-300 $\mathrm{K}$ temperature range. At room temperature, the agreement is excellent in both cases. The difference in loss from the single crystal to the polycrystalline is well explained in terms of the difference between the respective values of the linewidth. Thus, the main conclusion to be drawn is that the theory by Sparks et $a l .,{ }^{12}$ together with the measured values of the linewidth, gives a unified explanation of the microwave losses in both single crystal sapphire and polycrystalline alumina. 


\section{ACKNOWLEDGMENTS}

Thanks are due to Pablo Etchegoin, Jerzy Krupka, and Andrew Phillips for helpful discussions.

${ }^{1}$ Lord Rayleigh, Philos. Mag., Suppl. 43, 125 (1897).

${ }^{2}$ P. Debye, Ann. Phys. (Leipzig) 30, 57 (1909).

${ }^{3}$ R. D. Richtmyer, J. Appl. Phys. 10, 391 (1939).

${ }^{4}$ H. Rubens and G. Hertz, Berlin. Ber. 14, 256 (1912).

${ }^{5}$ P. P. Ewald, Naturwissenschaften 10, 1057 (1922).

${ }^{6}$ V. L. Gurevich, Sov. Phys. Solid State 21, 1993 (1979).

${ }^{7}$ V. L. Gurevich and A. K. Tagantsev, Sov. Phys. JETP 64, 142 (1987).

${ }^{8}$ V. L. Gurevich and A. K. Tagantsev, Adv. Phys. 40, 719 (1991).

${ }^{9}$ C. Herring, Phys. Rev. 52, 361 (1937).

${ }^{10}$ C. Herring, Phys. Rev. 52, 365 (1937).

${ }^{11}$ C. Herring, Phys. Rev. 95, 954 (1954).

${ }^{12}$ M. Sparks, D. F. King, and D. L. Mills, Phys. Rev. B 26, 6987 (1981).

${ }^{13}$ R. Stolen and L. Dransfeld, Phys. Rev. 139, 4A (1965).

${ }^{14}$ Eldridge, Phys. Rev. B 6, 1510 (1972).
${ }^{15}$ N. McN. Alford, S. Penn, A. Templeton, and X. Wang, J. Supercond. 10, 467 (1997).

${ }^{16}$ N. McN. Alford, J. Breeze, X. Wang, S. J. Penn, S. Dalla, S. J. Webb, N. Ljepojevic, and X. Aupi, J. Eur. Ceram. Soc. 21, 2605 (2001).

${ }^{17}$ E. H. Bogardus, J. Appl. Phys. 36, 2504 (1965).

${ }^{18}$ T. P. Srinivasan and J. Glourium Arulraj, J. Appl. Crystallogr. 24, 175 (1991).

${ }^{19}$ D. Kajfez and P. Guillon, Dielectric Resonator, 1st ed. (Artech House, Deham, MA, 1986).

${ }^{20}$ K. R. Subbaswamy and D. L. Mills, Phys. Rev. B 33, 4213 (1986).

${ }^{21}$ J. Wu and G. Ceder, Appl. Phys. 89, 5630 (2001).

${ }^{22}$ H. Bilz and W. Kress, Phonon Dispersion Relations in Insulators (Springer, New York, 1979).

${ }^{23}$ P. K. Govind and F. J. Fraikor, J. Appl. Phys. 43, 2448 (1972).

${ }^{24}$ H. Bialas and H. J. Stolz, Z. Phys. B 21, 319 (1975).

${ }^{25}$ J. Fontanella, C. Andeen, and D. Schuele, J. Appl. Phys. 45, 2852 (1974).

${ }^{26}$ G. Deinzer, G. Birner, and D. Strauch, Phys. Rev. B 67, 144304 (2003).

${ }^{27}$ A. Gobel, D. T. Wang, M. Cardona, L. Pintschovius, W. Reichardt, J. Kulda, N. M. Pyka, K. Itoh, and E. E. Haller, Phys. Rev. B 58, 10510 (1998).

\footnotetext{
The values for the fitting parameters for the linewidth gamma in $\mathrm{MgO}$ were wrong due to an arithmetic error in which 2 pi was inadvertently omitted in eq. 9. Where gamma $=10 \mathrm{~cm}-1$ this should read $2 \mathrm{~cm}-1$, where gamma $=46 \mathrm{~cm}-1$ this should read $10 \mathrm{~cm}-1$ and where gamma $=23$ $\mathrm{cm}-1$ this should read $5 \mathrm{~cm}-1$. These changes do not affect the experimental results or the conclusions.
} 